\title{
Exploring Cross-Channel Texture Correlation for Color Texture Classification
}

\author{
Xianbiao Qi ${ }^{1}$ \\ qixianbiao@gmail.com \\ Yu Qiao ${ }^{2}$ \\ yu.qiao@siat.ac.cn \\ Chun-Guang Li ${ }^{1}$ \\ lichunguang@bupt.edu.cn \\ Jun Guo ${ }^{1}$ \\ guojun@bupt.edu.cn
}

${ }^{1}$ Beijing University of Posts and Telecommunications, P.R. China

${ }^{2}$ Shenzhen Key Lab of CVPR, Shenzhen Institute of Advanced Technology, P.R. China

\begin{abstract}
This paper proposes a novel approach to encode cross-channel texture correlation for color texture classification task. Firstly, we quantitatively study the correlation between different color channels using Local Binary Pattern (LBP) as the texture descriptor and using Shannon's information theory to measure the correlation. We find that $(R, G)$ channel pair exhibits stronger correlation than $(R, B)$ and $(G, B)$ channel pairs. Secondly, we propose a novel descriptor to encode the cross-channel texture correlation. The proposed descriptor can capture well the relative variance of texture patterns between different channels. Meanwhile, our descriptor is computationally efficient and robust to image rotation. We conduct extensive experiments on four challenging color texture databases to validate the effectiveness of the proposed approach. The experimental results show that the proposed approach significantly outperforms its mostly relevant counterpart (Multichannel color LBP), and achieves the state-of-the-art performance.
\end{abstract}

\section{Introduction}

Color and Texture are two important aspects of natural images. It is widely recognized that they provide strong complementary cues to each other in a lot of computer vision applications, such as object recognition [21, 26], flower recognition [14, 19], texture classification [18], material recognition [10,23], content-based image retrieval, color texture segmentation and many more.

Recently, Local Binary Pattern (LBP) descriptor [15] and its variants [6, 7, 8, 16, 19, 30] have been widely applied on texture relevant tasks due to their computational efficiency and great texture discriminative ability. In [16], Timo et al. systematically introduced the LBP operator and its several variants including uniform LBP and rotation invariant LBP. Since then, LBP has been widely applied to a lot of tasks, such as face recognition, image retrieval, and many more [17]. Recently, in [7], Guo et al. proposed a completed LBP operator, which further enriches the original LBP feature. 


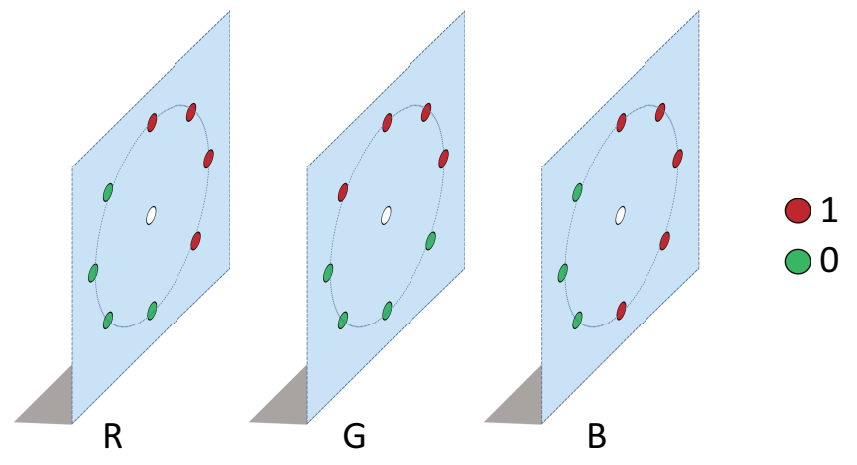

Figure 1: An illustration of LBPs in RGB channels.

The simplest and most direct method to incorporate color cue to the texture classification task is to extract LBP histograms from each color channel, and then concatenate the histograms into a final image representation. In the literature, this method has been used in several works [12, 31]. In [12], Maenpaa et al. used color LBPs (different color space) for texture classification, and in [31], Zhu et al. proposed to use color LBPs for object recognition.

However, the above color and texture fusing approach just treats each channel independently, and ignores the cross-channel texture correlation information. In fact, there exists strong correlation between texture patterns of different color channels. Such a correlation reflects the common character and the difference between patterns from different channels. Fig. 1 shows a typical structure in RGB color channels. The LBP sequences in RGB channels are greatly correlative to each other. Encoding the correlation effectively can greatly boost the discriminative ability of the feature.

In this paper, we propose a novel method to encode the cross-channel texture correlation to conduct color texture classification task. Firstly, we quantitatively study the texture correlation between different color channels using LBP as texture descriptor and using the Shannon's information entropy as correlation measurement. Based on the study, we find that $(\mathrm{R}, \mathrm{G})$ channel pair have stronger texture correlation than $(\mathrm{R}, \mathrm{B})$ and $(\mathrm{G}, \mathrm{B})$ channel pairs. Secondly, we propose a novel approach to encode the cross-channel texture correlation. The proposed method captures well the relative variance of the texture patterns between different channels. In practice, we divide three color channels into three pairs and encode one pair each time. The histograms of three pairs are finally concatenated into a final image representation. The proposed method is computationally efficient and robust to image rotation. Its computational cost is similar to the classical multi-channel color LBP. The novel encoding strategy of the proposed method can guarantee rotation invariance.

\section{Cross-Channel Texture Correlation}

\subsection{Preliminaries of LBP}

The LBP descriptor was firstly proposed by Ojala et al. [16] to depict pixel-wise texture information. Each LBP pattern depicts one kind of local structure of natural images, such 
as edge, contour, flat region and so on. For each pixel in an image, its LBP pattern can be calculated as follows:

$$
\operatorname{LBP}(n, r \mid c)=\sum_{k=0}^{n-1} s\left(g_{k}-g_{c}\right) 2^{k}, \quad s(x)= \begin{cases}1, & x \geq 0 \\ 0, & x<0,\end{cases}
$$

where $n$ is the number of neighbors and $r$ is the radius, $c$ is the central point, $g_{c}$ is the pixel value of the central pixel, and $g_{k}$ is the pixel value of its $k$-th neighbor.

Ojala et al. also reported that these patterns with very few spatial transitions depicted the fundamental properties of an image, and they called these patterns as "uniform patterns". In uniform patterns, the number of spatial transitions can be defined as follows:

$$
\Phi(\operatorname{LBP}(n, r \mid c))=\sum_{k=0}^{n-1}\left|s\left(g_{k}-g_{c}\right)-s\left(g_{k+1}-g_{c}\right)\right|,
$$

where $g_{n}$ equals to $g_{0}$. The uniform patterns are defined as these patterns with $\Phi(L B P(n, r \mid c)) \leq$ 2. For example, the LBP pattern "01111000" is a uniform pattern, and "00010110" is a nonuniform pattern.

The value of uniform LBP $\left(L B P^{u}\right)$ depends on the start point of the circular binary sequence. Defined on different start points, $L B P^{u}$ will have different values. For example, the uniform patterns of a circular sequence "00000001" defined on 1st or 2nd position are different. In this paper, we use $L B P^{u}(n, r \mid c, i)$ to denote the $L B P^{u}$ pattern defined on the start point $i$, where $0 \leq i \leq n-1$.

To achieve good robustness to image rotation, Ojala et al. introduced Rotation Invariant $\operatorname{LBP}\left(L B P^{r i}\right)$ and Rotation Invariant Uniform LBP $\left(L B P^{r i u}\right)$. $L B P^{r i u}$ can be defined as:

$$
L B P^{\text {riu }}(n, r \mid c)= \begin{cases}\sum_{k=0}^{n-1} s\left(g_{k}-g_{c}\right), & \Phi(\operatorname{LBP}(n, r \mid c)) \leq 2 \\ n+1, & \text { otherwise, }\end{cases}
$$

For the number of neighbors $n=8$, the LBP has $2^{8}=256$ patterns, in which there are 58 uniform patterns and 198 non-uniform patterns. Usually, all 198 non-uniform patterns are summarized into one pattern. Thus, $L B P^{u}$ has 59 patterns, and the $L B P^{r i u}$ has 10 patterns.

\subsection{Cross-Channel Texture Correlation}

Texture and color are two important and complementary information for natural images. They can help us to perceive and predict the material of the real world. Do texture patterns from different color channels have correlation? How strong is the correlation? And in which pair of channels, the texture correlation is stronger?

To answer these questions, we propose to use information entropy and mutual information to analyze the texture correlation. In information theory, entropy is used to measure the uncertainty of a random variable. In [22], Shannon defines the information entropy of a discrete random variable as:

$$
H(X)=\sum_{i=1}^{N}-p\left(x_{i}\right) \log _{b} p\left(x_{i}\right)
$$

where $p\left(x_{i}\right)$ is the probability of variable $X$ of $i$-th state, $N$ is the number of possible states, and $b$ is typically set to be 2 , e, or 10 . 
Similarly, the joint information entropy of two variables can be defined as:

$$
H(X, Y)=\sum_{x \in X} \sum_{y \in Y}-p(x, y) \log _{b} p(x, y),
$$

where $p(x, y)$ is the joint probability distribution function of $X$ and $Y$.

Mutual information is an effective method to characterize the correlation between variables. It provides an effective measure to characterize the mutual dependence of the two random variables. The mutual information can be defined as:

$$
I(X, Y)=\sum_{x \in X} \sum_{y \in Y} p(x, y) \log _{b}\left(\frac{p(x, y)}{p(x) p(y)}\right),
$$

where $p(x, y)$ is the joint probability distribution function of $X$ and $Y$, and $p(x)$ and $p(y)$ are the marginal probability distribution functions of $X$ and $Y$ respectively. The mutual information can also be denoted as $I(X, Y)=H(X)+H(Y)-H(X, Y)$. Larger $I(X, Y)$ means stronger correlation between $X$ and $Y$. In machine learning, mutual information has been used as a criterion for feature selection and feature transformations.

To quantitatively assess the correlation of texture patterns between different color channels, we use LBP as the texture descriptor. The statistics are conducted on about 30000 texture images, which come from the CUReT, KTH-TIPS, KTH-TIPS2a and ALOT databases. For each channel, we calculate a 256-dimensional LBP histogram. For a pair of two channel$\mathrm{s}$, we individually compute their LBP patterns in each channel, and then calculate a $256 \times 256$ co-occurrence histogram. We normalize the histograms with $L_{1}$ norm, then calculate the information entropy and mutual information according to Eq. 5 and Eq. 6. The statistical results are shown in Table 1.

Table 1: Texture Correlation between $(\mathrm{R}, \mathrm{G}),(\mathrm{R}, \mathrm{B})$, and $(\mathrm{G}, \mathrm{B})$.

\begin{tabular}{|c|c|c|c|c|}
\hline$(\mathrm{R}, \mathrm{G})$ & $\mathrm{H}(\mathrm{R})$ & $\mathrm{H}(\mathrm{G})$ & $\mathrm{H}(\mathrm{R}, \mathrm{G})$ & $\mathrm{I}(\mathrm{R}, \mathrm{G})$ \\
\hline Information Entropy & 4.37 & 4.35 & 6.87 & $\mathbf{1 . 8 4}$ \\
\hline (R, B) & $\mathrm{H}(\mathrm{R})$ & $\mathrm{H}(\mathrm{B})$ & $\mathrm{H}(\mathrm{R}, \mathrm{B})$ & $\mathrm{I}(\mathrm{R}, \mathrm{B})$ \\
\hline Information Entropy & 4.37 & 4.37 & 7.82 & 0.91 \\
\hline (G, B) & $\mathrm{H}(\mathrm{G})$ & $\mathrm{H}(\mathrm{B})$ & $\mathrm{H}(\mathrm{G}, \mathrm{B})$ & $\mathrm{I}(\mathrm{G}, \mathrm{B})$ \\
\hline Information Entropy & 4.35 & 4.37 & 8.05 & 0.67 \\
\hline
\end{tabular}

From Table 1, we have the following two key observations. Firstly, all channel pairs have strong correlation. Secondly, $(R, G)$ channels have stronger correlation than $(R, B)$ and $(G, B)$ since $I(R, G)$ is significantly larger than $I(R, B)$ and $I(G, B)$.

The significant correlation between different color channels reflects the common and different characteristics between patterns from different channels. Encoding the correlation may boost the discriminative power of features. Therefore, we propose a novel method to encode the cross-channel texture correlation below.

\subsection{Encoding Cross-Channel Texture Correlation}

To jointly encode pairwise color channels, we divide the three color channels into three pairs. Take RGB color space as an example, we separate them into $\{(R, G),(R, B),(G, B)\}$. Compared to joint encoding of all the three channels simultaneously, our strategy have two merits. Firstly, the dimension of joint encoding of two channels is much lower. Secondly, 


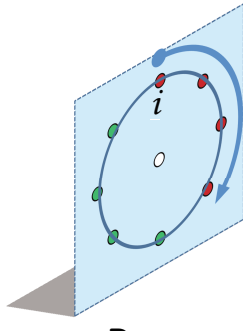

$\mathrm{R}$

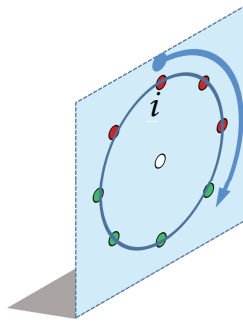

G

Figure 2: An illustration of encoding strategy between $(R, G)$ color channels.

this method has better robustness than the joint encoding of three color channels. If only one texture pattern out of three channels varies, our strategy can still achieve partial invariance.

Take $(R, G)$ channels as an example, as shown in Fig. 2, the Cross-Channel LBP $(C C L B P)$ can be defined as follows:

$$
C C L B P_{R, G}\left(n, r \mid c_{R}, c_{G}\right)=\left[\operatorname{LBP}_{R}^{r i u}\left(n, r \mid c_{R}\right), L B P_{G}^{u}\left(n, r \mid c_{G}, i\right)\right]_{c o},
$$

where

$$
i=\arg \max _{i}\left\{\operatorname{ROR}\left(L B P_{R}\left(n, r \mid c_{R}\right), i\right) \mid i=0,1, \ldots, n-1\right\},
$$

$c_{R}$ and $c_{G}$ are the central points of $R$ and $G$ channels respectively, $L B P_{R}^{r i u}\left(n, r \mid c_{R}\right)$ means the encoding of the $R$ channel with $L B P^{r i u}$, and $\left.L B P_{G}^{u}\left(n, r \mid c_{G}, i\right)\right)$ denotes to encode the $G$ channel using uniform LBP associated with the starting index of $i$, and $i$ is the index as defined in Eq. 8, which maximizes the LBP sequence in $R$ channel. $R O R(x, i)$ performs a circular bit-wise right shift on the P-bit number $x i$ times. Here, $[,]_{c o}$ is a co-occurrence operator. Our previous works $[19,20]$ have shown the effectiveness of co-occurrence.

As shown in Fig. 2, we have marked the position of $i$ on the figure. As indicated before, the uniform LBP pattern depends on the defined start point of the binary sequence. The position of $i$ will vary along with image rotation. Thus, the $L B P_{G}^{u}\left(n, r \mid c_{G}, i\right)$ is invariant to image rotation. Since $L B P_{R}^{r i u}\left(n, r \mid c_{R}\right)$ is invariant to image rotation, the encoding strategy of Eq. 7 is rotation invariant.

According to Eq. 7, the dimension of joint encoding of two channels is $10 \times 59=590$. In this paper, we use dense sampling to calculate the histogram. For each pixel in an image, we accumulate their joint patterns into the corresponding bin of histogram. Finally, we normalize the histogram feature with $L_{1}$ norm. The histograms of $(\mathrm{R}, \mathrm{B})$ and $(\mathrm{G}, \mathrm{B})$ can be built similarly. Thus, by concatenating these histograms of each channel pairs, we obtain the whole $C C L B P$ representation for an image as:

$$
C C L B P_{R, G, B}=\left[C C L B P_{R, G}, C C L B P_{R, B}, C C L B P_{G, B}\right] .
$$

in which the dimension of $C C L B P_{R, G, B}$ is $590 \times 3=1770$.

The $C C L B P$ has two merits. (1) $C C L B P$ feature is rotation invariant. We have detailly illustrated this merit using the Fig. 2 before. (2) The computational cost of CCLBP is low. Similar with the classical color LBP, we compute the LBP pattern in each channel, and then conduct the co-occurrence statistics according to Eq. 7. The cost for computing Eq. 7 is extremely low, and can be ignored. 


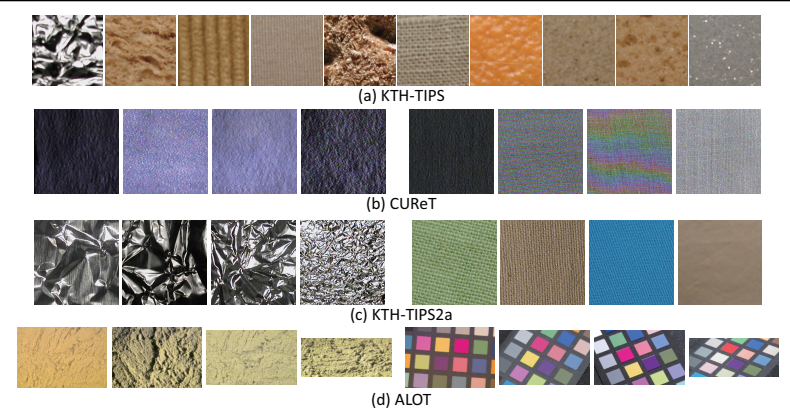

Figure 3: Samples of KTH-TIPS, CUReT, KTH-TIPS2a and ALOT.

\subsection{Image Representation based on Multi-Scale CCLBP}

In previous subsection, We have described the feature extraction process for a single scale. The multi-scale strategy [16] [11] has shown better performance than the single scale. Motivated by the work in [16] [11], we also adopt multi-scale $C C L B P$ for image representation and classification. Firstly, the $C C L B P$ features are extracted individually from each scale. Then, the features from all scales are concatenated into a final representation. Different from the classical multi-scale LBP (MS- $L B P$ ) that always uses larger neighbors at larger scale, we use just 8 neighbors for all scales to reduce the feature dimension. For instance, when using three scales, the classical MS- $L B P$ uses $\operatorname{LBP}(8,1), \operatorname{LBP}(16,2)$, and $\operatorname{LBP}(24,3)$. Instead, we use $\operatorname{LBP}(8,1), \operatorname{LBP}(8,2)$, and $\operatorname{LBP}(8,3)$.

\section{Experiments}

\subsection{Databases Introduction}

KTH-TIPS [9] contains 10 texture classes, with 81 images per class. The images are captured at 9 scales from three different illumination directions and three different poses. Note that this dataset has scale variance, but its scale changes continuously from 0.5 to 2 . We use 40 images per class for training and the rest for testing.

CUReT [5] is a widely used texture dataset. We use the same subset as [27]. The subset consists of 61 classes with 92 images per class. These images are captured under different illuminations from seven different viewing directions. The changes of viewpoint under different illumination greatly varies the texture appearance. Following [27], we use 46 samples per class for training and 46 for testing.

KTH-TIPS2 [2] is designed to study the generalization ability of material recognition methods to the new material instance. It includes 11 classes with 4 instances in each category. All 11 categories contain 4608 images in total. All instances are captured from varying viewing angles, lighting conditions and scales. Usually, 1, 2 or 3 instances from all 4 instances are used for training. The remained instances are used for testing.

ALOT [1] is a large color image collection. The database contains 250 classes with 100 images per class. The images are captured under varied viewing angles, illumination angle and illumination color. In addition, these images are captured using four different cameras. 
In this paper, we use 25 images from one camera for training and the rest images from the other three cameras for testing. Some samples from each dataset are shown in Fig. 3.

Classifier. To conduct the classification experiments, we adopt SVM with $\chi^{2}$ kernel. It has been shown in [3] that kernel SVM shows better performance than nearest neighbor classifier. After applying L1 normalization on features $X$ and $Y$, the similarity kernel can be denoted $S(X, Y)=\sum_{i=1}^{N} \frac{2 X_{i} \times Y_{i}}{X_{i}+Y_{i}}$. In this paper, we use Vlfeat [28] and Libsvm [4] for computing the kernel and conducting the classification. In our experiments, we find that for KTH-TIPS and CUReT, HSV color space works well and for KTH-TIPS2a and ALOT, RGB color space works well. Thus, we use HSV for KTH-TIPS and CUReT and use RGB color space for KTH-TIPS2a and ALOT.

We divide the experimental evaluation into two parts. In the first part, we validate the effectiveness of the proposed method by comparing it with its direct counter-part method. In the second part, we compare the proposed method with several state-of-the-art methods.

\subsection{Evaluation of the Proposed Method}

The effectiveness of Cross-Channel Texture Correlation. Here, we conduct experiments to validate the effectiveness of the proposed CCLBP by comparing it with gray-scale LBP and color LBP. We compare these three features on single scale. The scales $(8,1)$ and $(8,2)$ are individually used. We summarize the experimental results in Table 2.

Table 2: Comparison (\%) of $L B P^{\text {riu }}$, Color $L B P^{\text {riu }}, C C L B P$ on KTH-TIPS, CUReT.

\begin{tabular}{|c|c|c|c|c|c|c|}
\hline & \multicolumn{3}{|c|}{$(8,1)$} & \multicolumn{3}{c|}{$(8,2)$} \\
\hline Datasets & $L B P^{\text {riu }}$ & Color $L B P^{\text {riu }}$ & CCLBP & $L B P^{\text {riu }}$ & Color $L B P^{\text {riu }}$ & CCLBP \\
\hline KTH-TIPS(40) & 80.21 & 91.51 & $\mathbf{9 9 . 0 0}$ & 82.74 & 91.06 & $\mathbf{9 8 . 9 3}$ \\
\hline CUReT(46) & 60.91 & 82.77 & $\mathbf{9 8 . 6 8}$ & 64.95 & 84.38 & $\mathbf{9 8 . 8 9}$ \\
\hline
\end{tabular}

According to Table 2, it is apparent that the proposed CCLBP achieves significant improvement compared to the gray-scale LBP and the classical color LBP. For instance, on CUReT, using LBP $(8,1)$, gray-scale LBP $\left(L B P^{\text {riu }}\right)$ gets $60.91 \%$ and color LBP (color $L B P^{\text {riu }}$ ) obtains $82.77 \%$, but $C C L B P$ achieves $98.68 \%$ that improves gray-scale by about $38 \%$ and color LBP by about $16 \%$. The improvement well validates the effectiveness of CCLBP on capturing the cross-channels correlation.

Table 3: Performance evaluation (\%) of CCLBP on different scales.

\begin{tabular}{|c|c|c|c|c|c|}
\hline Databases & KTH-TIPS & CUReT & \multicolumn{3}{|c|}{ KTH-TIPS2a } \\
\hline Setting & 40 & 46 & 1 & 2 & 3 \\
\hline$(8,1)$ & 99.00 & 98.68 & 63.20 & 70.61 & 74.22 \\
$(8,2)$ & 98.93 & 98.89 & 64.89 & 71.16 & 74.83 \\
$(8,3)$ & 98.65 & 98.84 & 66.28 & 72.26 & 74.51 \\
$(8,1)+(8,2)$ & 99.12 & 99.14 & 66.01 & 73.16 & 76.82 \\
$(8,1)+(8,3)$ & 99.00 & 98.68 & 66.77 & 73.45 & 76.27 \\
$(8,1)+(8,3)$ & 99.05 & 99.16 & $\mathbf{6 7 . 6 5}$ & 73.16 & 76.57 \\
$(8,1)+(8,2)+(8,3)$ & $\mathbf{9 9 . 1 6}$ & $\mathbf{9 9 . 2 9}$ & $\mathbf{6 7 . 2 1}$ & $\mathbf{7 4 . 2 5}$ & $\mathbf{7 7 . 3 9}$ \\
\hline
\end{tabular}

The Influence of Multi-Scale. We also evaluate the influence of multi-scale strategy. Here, we use three scales $((8,1)$ and $(8,2)$ are $(8,3))$. The experiments are conducted on three databases including KTH-TIPS, CUReT and KTH-TIPS2a.

According to the experimental results in Table 3, we have the following two observations. (1) single-scale CCLBP has achieved great performance. For instance, CCLBP with (8, 
1) achieves $98.68 \%$ on KTH-TIPS and $99.00 \%$ on CUReT. (2) multi-scale CCLBP further improves the single scale $C C L B P$. For instance, on KTH-TIPS2a, multi-scale $C C L B P$ (using three scales) greatly outperforms any single scale $C C L B P$.

\subsection{Comparison with State-of-art Methods}

Experiments on KTH-TIPS and CUReT. Here, we conduct experiments on KTH-TIPS and CUReT. We report averaged performance over 100 random trials.

Table 4: Performance (\%) on KTH-TIPS and CUReT Database

\begin{tabular}{|c|c|c|c|c|c|}
\hline Methods & KTH-TIPS & CUReT & Methods & KTH-TIPS & CUReT \\
\hline Zhang et al. [29] & 96.1 & 95.3 & MS-LBP [16] & 94.1 & 91.7 \\
\hline Nguyen et al. [13] & 95.7 & - & Color MS-LBP & 95.9 & 94.9 \\
\hline VZ-patch [27] & 92.4 & 98.0 & PRI-CoLBP [19] & 98.3 & 98.6 \\
\hline Caputo et al. [3] & 94.8 & 98.0 & MS-CCLBP & $\mathbf{9 9 . 2}$ & $\mathbf{9 9 . 3}$ \\
\hline
\end{tabular}

From Table 4, we have three observations. Firstly, Color MS- $L B P$ and $C C L B P$ achieve better performance than gray-scale MS- $L B P$. This observation shows that color information is effective on both datasets. Secondly, CCLBP greatly outperforms Color MS- $L B P$. This fully validates the effectiveness of the proposed $C C L B P$ on depicting the cross-channel texture correlation. Finally, compared to several state-of-the-art methods, such as bag-of-sift [29], PRI-CoLBP [19], our CCLBP achieves better performance on both datasets.

Experiments on KTH-TIPS2a. KTH-TIPS2a has 11 categories with four instances in each category. Usually, several among four instances are used for training and the rest for testing. The evaluation method between [10] and [24] is different. For the work [10], when they use three instances per class for training, they repeat the random selection for each category. But for the work [24], they use the pre-selected three instances for all categories. It means that for the work [24], when 1th, 2th and 3th instances are selected for training, all categories use their corresponding 1 th, 2 th and 3 th instances for training. To fairly compare our work with them, we report two kinds of results.

Table 5: Performance (\%) on KTH-TIPS2a

\begin{tabular}{|c|c|c|c|c|}
\hline \multicolumn{5}{|c|}{ Comparison with LHS using split 1 } \\
\hline Setting & Dense SIFT [10] & MS-LBP [10] & MS-LBP+Color [10] & MS-CCLBP \\
\hline 1 & $45.5( \pm 3.6)$ & $59.1( \pm 3.7)$ & $61.4( \pm 2.8)$ & $\mathbf{6 7 . 7}( \pm 3.4)$ \\
\hline 2 & $52.3( \pm 2.3)$ & $65.8( \pm 1.4)$ & $70.4( \pm 0.7)$ & $\mathbf{7 3 . 2}( \pm 3.0)$ \\
\hline 3 & $56.4( \pm 2.6)$ & $70.7( \pm 3.6)$ & $73.1( \pm 4.6)$ & $\mathbf{7 4 . 8}( \pm 4.1)$ \\
\hline \multicolumn{5}{|c|}{ Comparison with LHS using split 2 } \\
\hline Setting & LBP $[16]$ & LTP $[25]$ & LHS $[24]$ & MS-CCLBP \\
\hline 3 & $69.8( \pm 6.7)$ & $71.3( \pm 6.3)$ & $73.0( \pm 4.7)$ & $\mathbf{7 7 . 4}( \pm 6.7)$ \\
\hline
\end{tabular}

Table 5 shows the performance of our method along with existing methods. Using the same evaluation method with [10], our approach significantly outperforms MS- $L B P$ for $8.6 \%$ and $3.9 \%$ using one or three instances for training respectively. Similarly, using the same evaluation method with [24], our method greatly outperforms their reported result (73.0\%). Meanwhile, our method also outperforms LBP, LTP.

From Table 5, we also find that our method has similar variance with the compared works under the same evaluation method. For instance, using the evaluation in [24], the variance of our method is $(6.7 \%)$, which is similar with LBP (6.7\%) and LTP $(6.3 \%)$. 
Experiments on ALOT. To systematically compare the CCLBP with gray-sacle and color LBP on ALOT, we compare them in single scale and multiple scales. For single scale, we use $\operatorname{LBP}(8,1)$. Similarly, color LBP and $C C L B P$ also use $(8,1)$. gray and color MS-LBP use $(8,1),(16,2)$ and $(24,3)$, but our MS-CCLBP just use $(8,1),(8,2)$ and $(8,3)$. In practice, our $C C L B P$ has lower computational cost. The results are shown in Table 6.

Table 6: Classification Accuracy (\%) of LBP, Color LBP and CCLBP on ALOT Dataset.

\begin{tabular}{|c|c|c|c|c|c|c|}
\hline Setting & \multicolumn{3}{|c|}{ Single Scale $(8,1)$} & \multicolumn{3}{c|}{ Multiple Scales } \\
\hline Methods & LBP & Color LBP & CCLBP & MS-LBP & Color MS-LBP & MS-CCLBP \\
\hline Camera 1 & 22.38 & 38.60 & $\mathbf{6 0 . 2 9}$ & 53.61 & 64.94 & $\mathbf{7 4 . 1 7}$ \\
\hline Camera 2 & 30.87 & 51.50 & $\mathbf{7 0 . 5 2}$ & 69.62 & 79.10 & $\mathbf{8 5 . 5 6}$ \\
\hline Camera 3 & 11.87 & 19.56 & $\mathbf{3 5 . 5 5}$ & 42.81 & 53.73 & $\mathbf{6 0 . 2 9}$ \\
\hline Camera 4 & 31.87 & 50.49 & $\mathbf{7 0 . 6 9}$ & 69.07 & 78.69 & $\mathbf{8 4 . 3 8}$ \\
\hline
\end{tabular}

For single scale, we can observe that color LBP and CCLBP significantly outperforms gray-scale LBP. The observation well validates that color information is effective on ALOT dataset. Meanwhile, we also find that $C C L B P$ achieves better performance than color LBP. This point shows that encoding the cross-channel texture correlation is effective for a lot of color texture classification tasks.

Multi-scale strategy greatly improve the recognition accuracy of their corresponding single-scale features. For instance, using the images from camera 1 for training, CCLBP gets $60.29 \%$, while MS-CCLBP using three scales gets $74.17 \%$ that improves single-scale $C C L B P$ by about $14 \%$. Meanwhile, MS-CCLBP also greatly outperforms its counterparts (MS- $L B P$ and Color MS- $L B P$ ). It should be noted that there exists strong image rotation on ALOT dataset, but the proposed approach still works well. This fact well demonstrates the robustness of the proposed method to image rotation.

\section{Conclusions}

This paper proposed a novel approach to encode cross-channel texture correlation for texture classification task. We quantitatively evaluated the cross-channel texture correlation of different color channels using LBP as the texture descriptor and using Shannon's information entropy as measurement of correlation. Our study indicates that $(R, G)$ channel pair has stronger correlation than $(\mathrm{R}, \mathrm{B})$ and $(\mathrm{G}, \mathrm{B})$ channel pairs. To capture such texture correlation, we propose a novel approach to encode the cross-channel texture correlation. The proposed approach captured well the relative variance of the texture patterns between different channels. Meanwhile, the proposed method is computationally efficient and robust to image rotation. Superior performance on four challenging color texture databases demonstrated the effectiveness of the proposed method.

\section{Acknowledge}

X. Qi, C.-G. Li and J. Guo are supported by the Natural Science Foundation of China (NSFC) under Grant nos. 61273217, 61175011, and 61005004. Y. Qiao is supported by NSFC (61002042), Shenzhen Basic Research Program, 100 Talents Programme of Chinese Academy of Sciences, and Guangdong Innovative Research Team Program (No.201001D0104648280). 


\section{References}

[1] Gertjan J Burghouts and Jan-Mark Geusebroek. Material-specific adaptation of color invariant features. Pattern Recognition Letters, 2009.

[2] Barbara Caputo, Eric Hayman, and P Mallikarjuna. Class-specific material categorisation. In $I C C V, 2005$.

[3] Barbara Caputo, Eric Hayman, Mario Fritz, and Jan-Olof Eklundh. Classifying materials in the real world. Image and Vision Computing, 2010.

[4] Chih-Chung Chang and Chih-Jen Lin. Libsvm: a library for support vector machines. ACM Transactions on Intelligent Systems and Technology (TIST), 2011.

[5] Kristin J Dana, Bram Van Ginneken, Shree K Nayar, and Jan J Koenderink. Reflectance and texture of real-world surfaces. ACM Transactions on Graphics (TOG), 1999.

[6] Yimo Guo, Guoying Zhao, and Matti Pietikäinen. Discriminative features for texture description. Pattern Recognition, 2012.

[7] Zhenhua Guo, Lei Zhang, and David Zhang. A completed modeling of local binary pattern operator for texture classification. TIP, 2010.

[8] Zhenhua Guo, Lei Zhang, and David Zhang. Rotation invariant texture classification using lbp variance (lbpv) with global matching. Pattern Recognition, 2010.

[9] Eric Hayman, Barbara Caputo, Mario Fritz, and Jan-Olof Eklundh. On the significance of real-world conditions for material classification. ECCV, 2004.

[10] Wenbin Li and Mario Fritz. Recognizing materials from virtual examples. In ECCV. 2012.

[11] Topi Mäenpää and Matti Pietikäinen. Multi-scale binary patterns for texture analysis. Image Analysis, 2003.

[12] Topi Mäenpää and Matti Pietikäinen. Classification with color and texture: jointly or separately? Pattern Recognition, 2004.

[13] Huu-Giao Nguyen, Ronan Fablet, and J-M Boucher. Visual textures as realizations of multivariate log-gaussian cox processes. In CVPR, 2011.

[14] M-E Nilsback and Andrew Zisserman. Automated flower classification over a large number of classes. In IEEE Sixth Indian Conference on Computer Vision, Graphics \& Image Processing, 2008.

[15] Timo Ojala, Matti Pietikäinen, and David Harwood. A comparative study of texture measures with classification based on featured distributions. Pattern recognition, 1996.

[16] Timo Ojala, Matti Pietikainen, and Topi Maenpaa. Multiresolution gray-scale and rotation invariant texture classification with local binary patterns. PAMI, 2002.

[17] M Pietikäinen, A Hadid, G Zhao, T Ahonen, M Pietikäinen, A Hadid, G Zhao, and T Ahonen. Computer vision using local binary patterns. 2011. 
[18] Matti Pietikainen, Topi Maenpaa, and Jaakko Viertola. Color texture classification with color histograms and local binary patterns. In Workshop on Texture Analysis in Machine Vision, 2002.

[19] Xianbiao Qi, Rong Xiao, Jun Guo, and Lei Zhang. Pairwise rotation invariant cooccurrence local binary pattern. In ECCV. 2012.

[20] Xianbiao Qi, Yi Lu, Shifeng Chen, Chun-Guang Li, and Jun Guo. Spatial cooccurrence of local intensity order for face recognition. In ICME workshop. 2013.

[21] F Shahbaz Khan, Joost van de Weijer, and Maria Vanrell. Top-down color attention for object recognition. In ICCV, 2009.

[22] Claude Elwood Shannon. The mathematical theory of communication, volume 117.

[23] Lavanya Sharan, Ce Liu, Ruth Rosenholtz, and Edward H Adelson. Recognizing materials using perceptually inspired features. IJCV, 2013.

[24] Gaurav Sharma, Sibt ul Hussain, and Frédéric Jurie. Local higher-order statistics (lhs) for texture categorization and facial analysis. In ECCV. 2012.

[25] Xiaoyang Tan and Bill Triggs. Enhanced local texture feature sets for face recognition under difficult lighting conditions. In Analysis and Modeling of Faces and Gestures. 2007.

[26] Koen EA Van De Sande, Theo Gevers, and Cees GM Snoek. Evaluating color descriptors for object and scene recognition. PAMI, 2010.

[27] Manik Varma and Andrew Zisserman. A statistical approach to material classification using image patch exemplars. PAMI, 2009.

[28] Andrea Vedaldi and Brian Fulkerson. Vlfeat: An open and portable library of computer vision algorithms. In ACM Multimedia, 2010.

[29] Jianguo Zhang, Marcin Marszałek, Svetlana Lazebnik, and Cordelia Schmid. Local features and kernels for classification of texture and object categories: A comprehensive study. IJCV, 2007.

[30] Lin Zhang, Lei Zhang, Zhenhua Guo, and David Zhang. Monogenic-lbp: a new approach for rotation invariant texture classification. In ICIP, 2010.

[31] Chao Zhu, C-E Bichot, and Liming Chen. Multi-scale color local binary patterns for visual object classes recognition. In ICPR, 2010. 